\title{
Observation of two-dimensional longitudinal-transverse correlations in an electron beam by laser-electron interactions
}

\author{
G. Angelova and V. Ziemann \\ Department of Physics and Astronomy, Uppsala University, 75121 Uppsala, Sweden \\ A. Meseck \\ BESSY, Albert-Einstein-Strasse 15, 12489 Berlin, Germany \\ P. Salén, P. van der Meulen, M. Hamberg, and M. Larsson \\ Department of Physics, Stockholm University, AlbaNova University Center, 10691 Stockholm, Sweden \\ J. Bödewadt, S. Khan, and A. Winter \\ Universitat Hamburg, Institute of Experimental Physics, Luruper Chaussee 149, 22761 Hamburg, Germany \\ H. Schlarb, F. Löhl, E. Saldin, E. Schneidmiller, and M. Yurkov \\ DESY, Notkestraße 85, 22607 Hamburg, Germany
}

(Received 17 December 2007; published 24 July 2008)

\begin{abstract}
During the preparatory work for the optical-replica synthesizer experiment in the free-electron laser FLASH at DESY, we were able to superimpose a short, approximately $200 \mathrm{fs}$ long pulse from a frequencydoubled mode-locked erbium laser with titanium-sapphire amplifier and an approximately $20 \mathrm{ps}$ long electron bunch in an undulator. This induces an energy modulation in a longitudinal slice of the electron bunch. A magnetic chicane downstream of the undulator converts the energy modulation into a density modulation within the slice that causes the emission of coherent optical transition radiation from a silvercoated silicon screen. Varying the relative timing between electron and laser, we use a camera to record two-dimensional images of the slices as a function of the longitudinal position within the electron bunch.
\end{abstract}

PACS numbers: 29.20.Ej, 29.27.Fh

\section{INTRODUCTION}

In the optical-replica synthesizer (ORS) experiment, we intend to measure the longitudinal profile of ultrarelativistic electron bunches in the free-electron laser FLASH at DESY by creating a coherent optical pulse with the same longitudinal profile as the electron bunch [1]. This is achieved by energy modulating the electron bunch with a short laser pulse in an undulator (the "modulator") which produces spontaneous radiation at the same wavelength and with the same polarization state as the laser. The energy modulation is subsequently converted into a density modulation in a magnetic chicane. In a second undulator (the "radiator"), tuned to the same wavelength, the microbunched electrons will emit a coherent pulse that contains all necessary information to extract the longitudinal electron bunch profile. The electron bunch profile can be obtained from the optical pulse using well-established frequency-resolved optical gating (FROG) techniques [2].

During commissioning, we first addressed the reliable operation of all subsystems and then tried to achieve spatial and temporal overlap of the laser pulse and the electron bunch. During these experiments we were able to selectively excite longitudinal slices of the electron bunch with a short laser pulse and cause this slice to emit coherent transition radiation [3] when hitting an optical transition radiation (OTR) screen. Varying the relative electron-laser timing yields information about longitudinal-transverse correlations in the electron bunch.

Laser-induced energy modulation can also be used to generate short x-ray pulses ("femto-slicing" [4]), where off-energy electrons are transversely displaced in order to extract a short pulse of synchrotron radiation. In this case, the energy-dependent path length differences are much larger and microbunching does not occur. On the other hand, a dip in the longitudinal charge distribution is produced, which also gives rise to coherent radiation. In contrast to ORS, this radiation is not at the laser wavelength, but has a broad spectrum at wavelengths comparable to the size of the dip (about $0.1 \mathrm{~mm}$ ) and longer. This $\mathrm{THz}$ radiation component has also been used to optimize the electron-laser overlap [5-7], but yields no information on the transverse bunch properties.

In the subsequent sections of this report, we first describe the setup of the ORS and then discuss the experiments regarding the overlap and the correlations.

\section{OPTICAL-REPLICA SETUP}

The hardware for the experiment, comprising a laser system, laser transport and diagnostic, two undulators, and a magnetic chicane has been completely installed in FLASH since the summer of 2007. Figure 1 shows a schematic overview of the relevant components where 


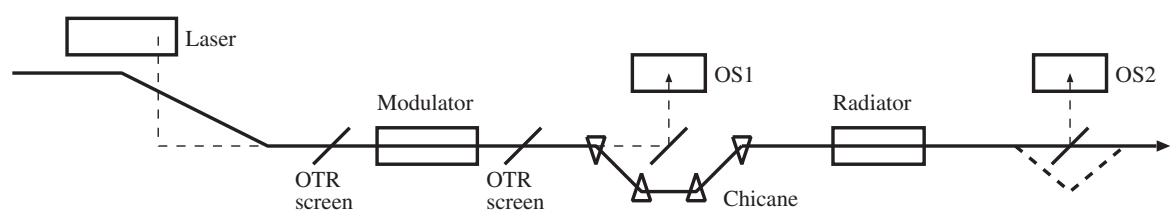

FIG. 1. The schematic setup of the experiment.

the electron beam is going from left to right. The laser system which is located in a laser laboratory outside the accelerator tunnel comprises a fiber oscillator [8] operating at $1550 \mathrm{~nm}$ that is phase locked to the radio-frequency (rf) system of the accelerator. After amplification and frequency doubling, the pulses are fed into a regenerative titanium-sapphire amplifier (Clark-MXR CPA-2001), based on chirped-pulse amplification and pumped by a Nd:YAG laser. This system, initially prepared at Stockholm University, delivers pulses with a center wavelength of $772 \mathrm{~nm}$, up to $1 \mathrm{~mJ}$ energy per pulse and a fullwidth at half-maximum (FWHM) pulse length of presently 200 fs. For optical-replica operation, the laser system compressor will be retuned to provide about 2 ps pulses. The laser pulses are sent to the accelerator tunnel through a $12 \mathrm{~m}$ long transport system with remotely controlled mirrors and a telescope. This allows us to control the laser waist position and size in the laser-electron interaction zone inside the modulator located about $10 \mathrm{~m}$ downstream of the back-tangent window where the laser pulse enters the accelerator beam pipe. The window is located near the second dipole of a dog-leg chicane used for momentum collimation. The laser pulse copropagates with the electrons for about $16 \mathrm{~m}$ until it is extracted by a silver-coated silicon OTR screen installed in the middle of a four-magnet chicane and is diagnosed by a camera, photodiodes, and a power meter on the first optical station (OS1). The electrons continue to propagate through the radiator and pass a second optical station (OS2) where the light created at or reflected from a silver-coated silicon OTR screen is guided to an optical table where it is recorded by a Basler A331

TABLE I. The parameters used during the experiments.

\begin{tabular}{lccc}
\hline \hline Parameter & Value & Range & Unit \\
\hline Beam energy & 700 & $(450-1000)$ & $\mathrm{MeV}$ \\
Bunch charge & 1 & $(0.5-1.2)$ & $\mathrm{nC}$ \\
Average $\beta_{x}, \beta_{y}$ in modulator & $14.5,9.7$ & & $\mathrm{~m}$ \\
Undulator period length & 0.2 & & $\mathrm{~m}$ \\
Undulator periods & $5+2$ & & \\
Undulator peak field & 0.28 & $(0-0.45)$ & $\mathrm{T}$ \\
Undulator K value & 5.2 & $(0-8.4)$ & \\
Laser wavelength & 772 & & $\mathrm{~nm}$ \\
Laser pulse energy (at laser) & 0.7 & & $\mathrm{~mJ}$ \\
Laser pulse energy (at undulator) & 0.5 & & $\mathrm{~mJ}$ \\
Laser pulse length (FWHM) & 200 & $(150-3000)$ & $\mathrm{fs}$ \\
Rayleigh length in modulator & 6 & & $\mathrm{~m}$ \\
$R_{56}$ of chicane & 170 & $(0-300)$ & $\mu \mathrm{m}$ \\
\hline \hline
\end{tabular}

camera. The optical table also houses a power meter and the GRENOUILLE [9] which is a commercial FROG device that will be used to reconstruct the longitudinal electric field profile of the replica pulse. For the ORS experiments the electron beam will be directed around the OTR screen on OS2 by three steering magnets, as is shown by the dashed line in Fig. 1, but can also be directed onto the OTR screen by switching off the three steering magnets in order to observe the coherent radiation stemming from the microbunched beam [3].

The two undulators were designed, assembled, and tested by Scanditronix Magnet AB, Sweden. They have a length of $1450 \mathrm{~mm}$ and weight $480 \mathrm{~kg}$ and can be mounted horizontally or vertically. Their gap is $40 \mathrm{~mm}$ in order to accommodate the standard $38 \mathrm{~mm}$ vacuum pipe without having to break vacuum. The period length is $200 \mathrm{~mm}$ with 14 poles and 28 coils connected in 4 separate coil circuits. This allows adjusting the field integrals $I_{1}=\int_{0}^{L} B(z) \mathrm{d} z$ and $I_{2}=\int_{0}^{L} \int_{0}^{z} B\left(z^{\prime}\right) \mathrm{d} z^{\prime} \mathrm{d} z$ by controlling the end coils in the $1 / 4,-3 / 4,+1$ pattern. The first and the last coil (with $1 / 4$-excitation) have independent power supplies and the

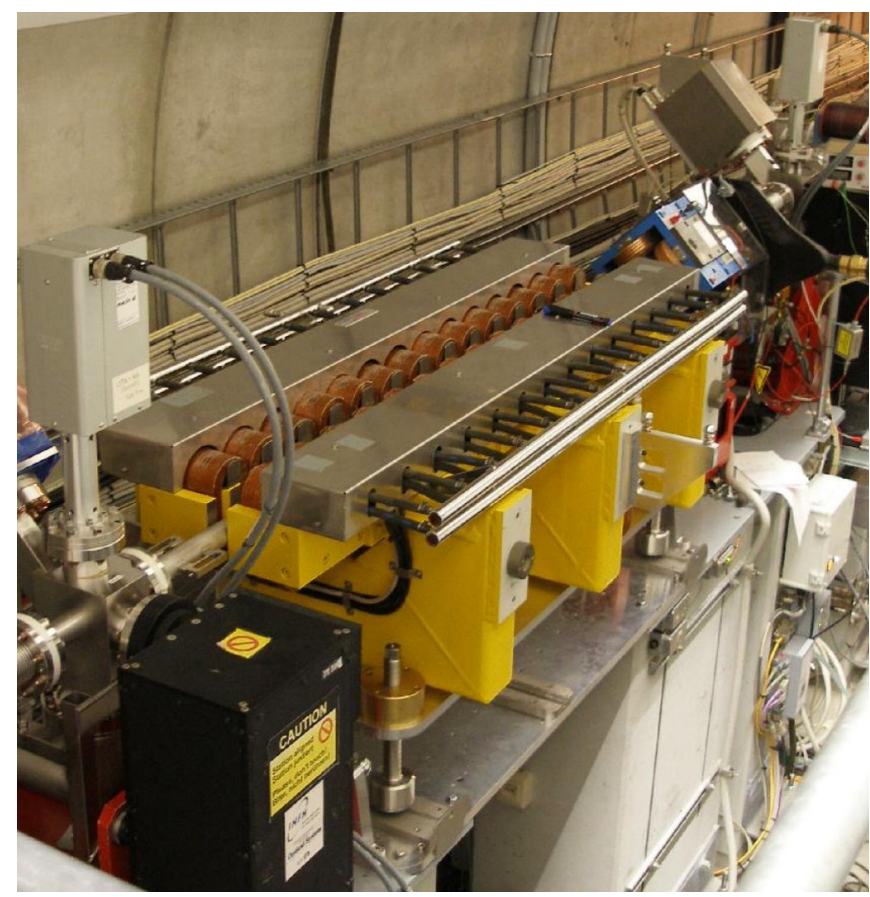

FIG. 2. (Color) The modulator, an electromagnetic undulator in which vertically polarized laser pulses interact with electron bunches, causing a periodic modulation of the electron energy. 
second and the 13th coil (with 3/4-excitation) are powered in series by another power supply. The set values of the power supplies were determined in bench measurements of the undulators and fine adjusted with beam early in commissioning runs. Finally, the ten central coils are connected in series to a fourth power supply capable of achieving a maximum magnetic field of $0.45 \mathrm{~T}$. The modulator, shown in Fig. 2, is mounted such that the beam performs vertical oscillations while the radiator causes horizontal beam oscillations. This allows one to separate the vertically polarized laser pulse from the horizontally polarized replica pulse generated in the radiator. The chicane is located between the undulators and consists of four steering dipole magnets that allow a maximum excursion of the beam of $15 \mathrm{~mm}$, thereby producing an $R_{56}$ of $300 \mu \mathrm{m}$.

\section{LASER-ELECTRON INTERACTION}

One of the key issues for the ORS experiment is to achieve spatial and temporal overlap of electrons and laser pulses in the modulator. This is a particular challenge because of the 2-ps long laser pulse and the very short electron bunch length, which can include a high current spike with shorter than $100 \mathrm{fs}$ duration. This is about one to two orders magnitude shorter than can be detected on available oscilloscopes. In order to facilitate finding the overlap between electrons and laser pulse during initial commissioning, we operated with an uncompressed electron bunch and kept the laser as short as possible at about 200 fs FWHM. This configuration relaxes the timing tolerance while increasing the modulation exerted by the laser pulse onto the electron bunch. The laser and accelerator
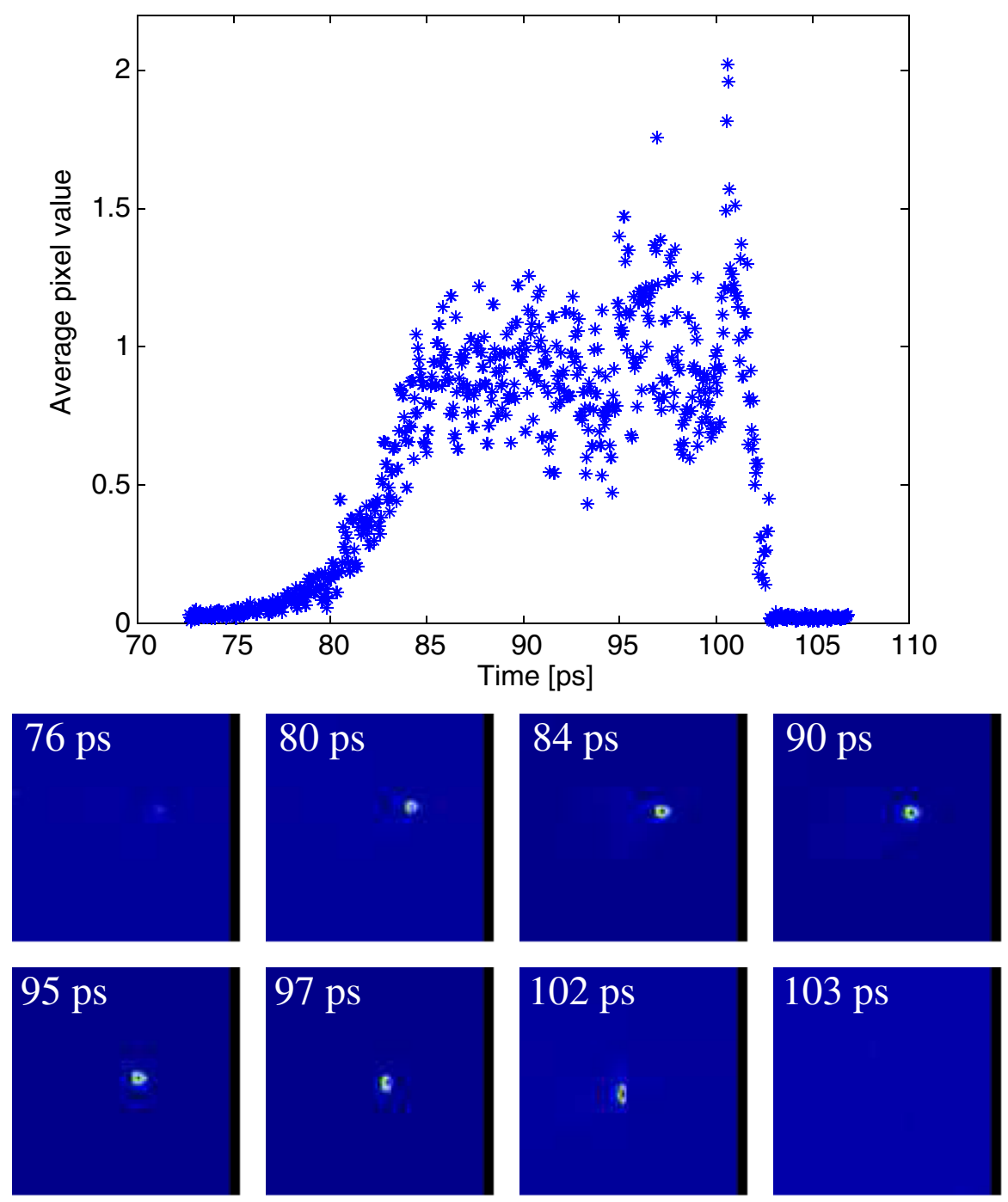

FIG. 3. (Color) A sequence of pictures (below) recorded on a camera on OS2 as the $200 \mathrm{fs}$ laser pulse samples different longitudinal slices of the electron bunch. The size of the area shown on the pictures is $5 \mathrm{~mm}$ by $5 \mathrm{~mm}$. The upper plot shows the average pixel value in a small region around the spot. It shows a clear enhancement as the laser passes the electrons from the tail of the electron bunch at the left towards the head of the bunch at the right. 
parameter settings we used during the experiments are listed in Table I.

The transverse overlap was achieved by observing the electron beam on OTR screens and the laser beam on diffuse aluminum screens located immediately upstream and downstream of the modulator and placing both beams on the same transverse position. The transverse rms electron beam size was about $0.3 \mathrm{~mm}$ and the rms laser beam size was about $0.6 \mathrm{~mm}$ limited by the installed lenses in the telescope which resulted in a Rayleigh length of $6 \mathrm{~m}$. Consequently, the transverse laser beam size only varies in the percent range over the length of the undulator. The initial, coarse temporal overlap was adjusted by observing the laser pulse and the spontaneous synchrotron radiation emitted by the modulator on a photodiode at OS1 using a fast oscilloscope while adjusting the phase of the laser oscillator relative to the accelerator rf. In this way an accuracy of about 100 to 200 ps could be achieved. For temporal fine-tuning we resorted to scanning a phase shifter that moves the rf reference of the servo loop that locks the laser oscillator [8] to the rf reference of the accelerator. The oscillator is phase locked to the rf system of FLASH with a timing jitter of approximately $50 \mathrm{fs}$. The jitter with respect to the electron bunches in FLASH is on the order of up to $1 \mathrm{ps}$.

In the region where the electron bunch and the short laser pulse overlap in the modulator, a local modulation of the electron energy ensues if the magnetic field of the undulator is adjusted to fulfill the resonance condition. This is undemanding due to the large width of the resonance because of the small number of periods of the undulator. This local energy modulation has a modulation depth of about $1200 \mathrm{keV}$ or $\Delta p / p \approx 1.7 \times 10^{-3}$. It also has the same period as the laser wavelength and is transformed into a longitudinal density modulation in the chicane where the laser pulse is extracted in order to avoid excessive background radiation at OS2. The energy modulation of the slice is detected by passing the electron bunch through an OTR screen at OS2 where the modulated slice emits a light pulse at the modulation wavelength [3] and its harmonics. Initially we tried to observe the signal at the first harmonic, but despite extracting the bulk of the laser pulse at OS1 the signal on the camera at OS2 was still dominated by the laser pulse despite attempts to deflect it onto OS1 with a mirror. The OTR screen used to extract the laser pulse does not completely block the beam line and a small fraction of the seed laser pulse passes on the side. Moreover, the reflective layer on the screen is rather thin and part of the laser pulse passes through the mirror. We solved this problem by placing a BG39 optical filter in front of the OS2 camera to attenuate the radiation at the first harmonic and observed the radiation from the OTR screen at the second harmonic. Both the incoherent OTR and the light from the second harmonic caused by the density modulation can thus penetrate the filter but the coherent pulse is significantly enhanced compared to the incoherent OTR emitted by the remainder of the electron bunch. The light from the OTR screen is recorded by a camera triggered synchronously with the beam. In order to prevent saturation, we used a neutral density filter that reduced the intensity on the camera by a factor 100 . At the bottom of Fig. 3 we display a selection of pictures recorded while continuously scanning the relative timing between laser and electrons. The first picture at $76 \mathrm{ps}$ shows the first faint signal as the laser starts interacting with the electrons. As the laser pulse overlaps more with the electron pulse, the intensity increases and then stays roughly constant during the passage through the majority of the bunch. At $102 \mathrm{ps}$ it has reached the head of the bunch and at $103 \mathrm{ps}$ the laser pulse misses the electron bunch entirely. The top of Fig. 3 shows the average value of the pixels within a small region around the spot as a function of the relative timing between electrons and laser pulse. The signal is a clear signature of the laser-electron interaction and is used to find the temporal overlap.

One obvious feature of the sequence of pictures is that the coherently excited slice moves downwards and towards the left as the laser passes through the electron bunch. This means that the tail of the electron bunch has a different transverse position compared to the head of the bunch. Figure 4 displays the position of the peak of the coherent spot as a function of the relative timing between laser pulse and electron beam. The transverse position of the excited slice moves by up to $1 \mathrm{~mm}$ over the length of the bunch. The bunch thus has a parabolic shape such that the tail is bent away from the position of the head. This shows that detection of longitudinal-transverse correlations in two transverse dimensions simultaneously of ps long electron bunches is possible.

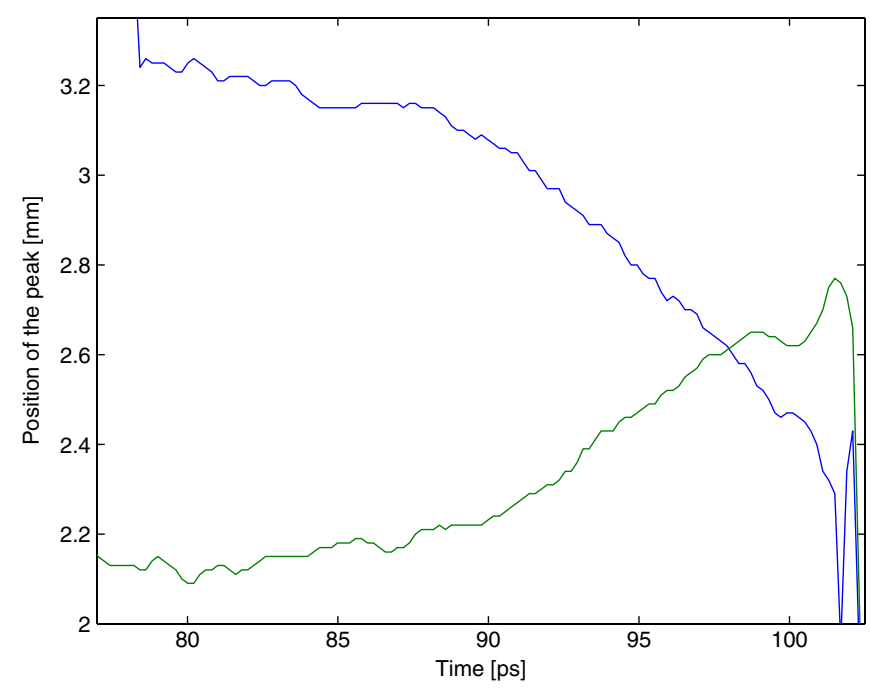

FIG. 4. (Color) Position of the horizontal (blue) and vertical (green) center of the coherently excited signal along the length of the electron bunch. The positions are measured from the topleft corner of the small images in Fig. 3. 


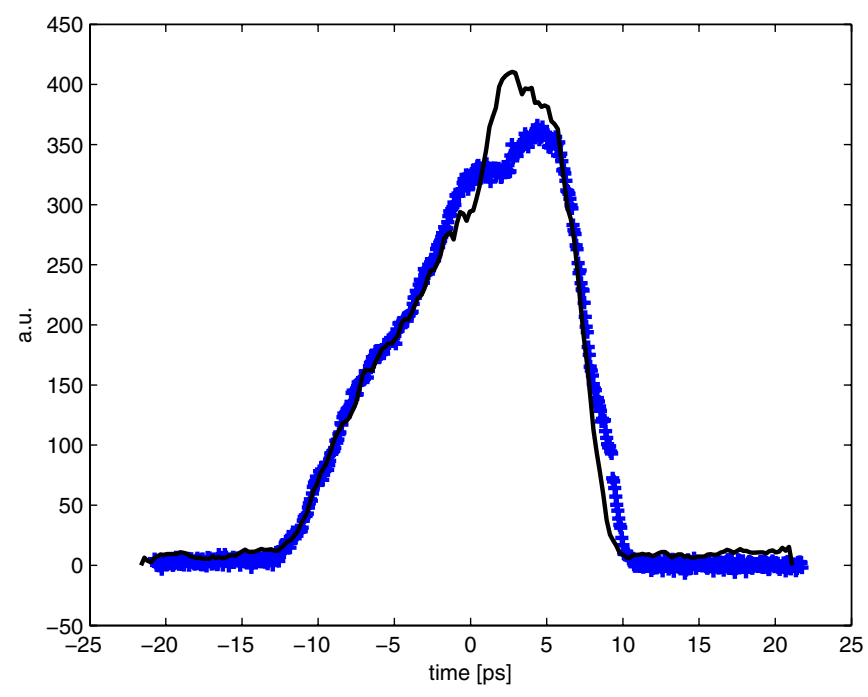

FIG. 5. (Color) Comparison of the longitudinal profile of the electron bunch measured with the transversely deflecting cavity LOLA (blue crosses) and the optical-replica synthesizer (black).

During another shift we measured the longitudinal profile of the electron bunch with a transversely deflecting cavity (LOLA) installed in FLASH [10] almost simultaneously with a scan of the relative timing of laser and electron bunch in the ORS. Also during these measurements we inserted a neutral density filter that reduces the intensity on the camera by a factor 100 in order to avoid saturation. Note that the signal recorded by the camera is a coherent signal and therefore quadratic in the local electron density in the bunch. Consequently, we have to take the square root of the ORS signal. We also shift the time axis of the ORS signal and adjust the vertical scale and finally smooth the signal with a 11-point averaging filter. The result is displayed in Fig. 5 which shows remarkable agreement of the bunch profiles determined by the two methods. We find that the curves agree especially well on the shoulders.

\section{DISCUSSION}

One key feature of the performed experiments was to reliably determine spatial and temporal overlap of electron bunch and laser pulse, which was successful and reproducible in consecutive shifts. The fact that the laser beam was rather wide and intense made this rather unproblematic. The other key feature is the ability to selectively affect a longitudinal slice of the electron bunch and then detect the transverse position on an OTR screen. We expect that this can be used to analyze longitudinal-transverse correlations of electron bunches.
The shortest laser pulses we used during the experiments were about $200 \mathrm{fs}$. If the laser pulses were even shorter, the relative jitter between electrons and laser became important. Conversely, placing the laser pulse at the head of the bunch where the variation of the electron bunch varies dramatically with the relative timing of the laser will present a means to quantify the jitter. In the present configuration, while passing the electrons through an OTR screen, only dedicated measurements are possible because the screen makes the beam unusable for other experiments. However, tuning the radiator onto the second harmonic and steering the electrons around the screen leaves the electron beam intact and may allow parasitic operation which seems feasible since we successfully tested the generation and detection of the coherent radiation from the radiator, both at the first and second harmonic, albeit not yet the replica generation.

\section{ACKNOWLEDGMENTS}

The continuous support from the technical groups at DESY and the University of Hamburg as well as the DESY management is essential for this experiment and is gratefully acknowledged. Furthermore we thank Jens Osterhoff, MPQ, Garching for discussions and help during the shifts. This work is supported by the UU-SU-KTH FEL-center.

[1] E. Saldin, E. Schneidmiller, and M. Yurkov, Nucl. Instrum. Methods Phys. Res., Sect. A 539, 499 (2005).

[2] R. Trebino, Frequency Resolved Optical Gating (Kluwer Academic, Boston, 2000).

[3] J. Rosenzweig, G. Travish, and A. Tremaine, Nucl. Instrum. Methods Phys. Res., Sect. A 365, 255 (1995).

[4] A. A. Zholents and M. S. Zolotorev, Phys. Rev. Lett. 76, 912 (1996).

[5] K. Holldack et al., Phys. Rev. ST Accel. Beams 8, 040704 (2005).

[6] J. M. Byrd et al., Phys. Rev. Lett. 96, 164801 (2006).

[7] A. Streun et al., in Proceedings of the 10th European Particle Accelerator Conference, Edinburgh, Scotland, 2006 (EPS-AG, Edinburgh, Scotland, 2006), p. 3427.

[8] A. Winter, P. Schmüser, F. Loehl, H. Schlarb, and W. Jalmuzna, in Proceedings of the 2007 Particle Accelerator Conference, Albuquerque, New Mexico, 2007 (IEEE, Albuquerque, New Mexico, 2007), p. 191.

[9] Swamp Optics, http://www.swampoptics.com.

[10] M. Röhrs, C. Gerth, and H. Schlarb, in Proceedings of the 2007 Particle Accelerator Conference, Albuquerque, New Mexico, 2007 (Ref. [8]). 